\title{
Signaling Pathways as Specific Pharmacologic Targets for Neuroendocrine Tumor Therapy: RET, PI3K, MEK, Growth Factors, and Notch
}

\author{
Yvette Carter Renata Jaskula-Sztul Herbert Chen Haggi Mazeh \\ Section of Endocrine Surgery, Department of Surgery, University of Wisconsin, Madison, Wisc., USA
}

\section{Key Words}

Neuroendocrine tumors $\cdot$ Carcinoid $\cdot$ Medullary thyroid cancer $\cdot$ Signaling pathways $\cdot$ RET $\cdot$ PI3K $\cdot$ MEK $\cdot$ Growth factors $\cdot$ Notch

\begin{abstract}
Neuroendocrine tumors are rare tumors with a common progenitor - the neural crest cell. Included in this category are pulmonary and gastrointestinal tract carcinoid tumors and medullary thyroid cancer. The majority of these tumors are sporadic in nature, however they can be hereditary. Medullary thyroid cancers can present sporadically, with other endocrine tumors, as in the complex of multiple endocrine neoplasias 1, 2A, or 2B, or as familial medullary thyroid cancer. These tumors can become evident at later stages, with metastases already present at the time of diagnosis. Despite the small size and rare incidence of gastrointestinal neuroendocrine (carcinoid) tumors, they can be debilitating when present. Their natural history presents as early lymph node and distant metastases, as well as symptoms of the carcinoid syndrome, which result from the overproduction and secretion of serotonin and somatostatin. As a consequence of their metastases, surgical resection is non-curative and hence there is a need for novel treatment strategies to address tumor burden and symptom control. There are multi-
\end{abstract}

ple intracellular pathways which can be targeted, either individually or in combination, to address these tumors. Here, we review some of the intracellular pathways, and identify some specific targets, which are vital to the generation and propagation of neuroendocrine tumorigenesis, and thus, can be the foci of novel drug therapies. We also elaborate on present pharmacological strategies and clinical trials involving these intracellular pathways.

Copyright $\odot 2012$ S. Karger AG, Basel

\section{Introduction}

Neuroendocrine tumors (NETs), though rare in incidence $(2-5$ per 100,000$)$, are of clinical significance due to their presentation and symptoms [1]. These tumors are often well differentiated, with indolent behaviors; however, some patients present late, with widespread metastatic disease. As a result of their common embryologic derivation from neural crest cells, these tumors can secrete a variety of substances, including: chromogranin A (CgA), serotonin or 5-hydroxytryptamine, synaptophysin, somatostatin, and neuron-specific enolase. Accordingly, patients may present with different isolated symptoms or in carcinoid syndrome resulting from overproduction and secretion of these bioactive products (right

\section{KARGER}

Fax +4161306 1234

E-Mail karger@karger.ch

www.karger.com
(C) 2012 S. Karger AG, Basel

0028-3835/13/0971-0057\$38.00/0

Accessible online at:

www.karger.com/nen
Haggi Mazeh, MD

Section of Endocrine Surgery, Department of Surgery

University of Wisconsin, K4/739 Clinical Science Center

600 Highland Avenue, Madison, WI 53792 (USA)

Tel. +1 608263 1387, E-Mail mazeh@ @urgery.wisc.edu 
heart valvular disease, congestive heart failure, flushing, and diarrhea) $[2,3]$. Gastrointestinal NETs frequently metastasize to the liver, and these are usually evident at the time of presentation, making curative resection less feasible. When surgical resection cannot offer cure, treatment with long-acting somatostatin analogs may decrease the progression and symptoms of the NETs as approximately $90 \%$ have somatostatin receptors $[4,5]$. Long-acting octreotide may increase the time to progression of both active and inactive metastatic midgut NETs, however this is a costly treatment with documented side effects and does not provide a therapeutic option to many patients $[5,6]$. The resistance of NETs to conventional chemotherapy and radiation therapy leads to the investigation of novel therapies targeting intracellular signaling pathways, as pertinent to the treatment of these debilitating tumors.

The intracellular pathway initiator, which plays a role in all neuroendocrine-derived tumors, is the rearranged during transfection (RET) oncogene. This well-studied oncogene can be a general, non-specific therapeutic target, as it activates three intracellular pathways: Ras/Raf/ mitogen-activated protein kinase kinase (MEK)/extracellular signal-related kinase (ERK), c-Jun N-terminal kinases (JNK), and phosphotidylinositol 3'-kinase (PI3KAkt) [7]. In addition, its inactivation results in caspasedependent apoptotic neuronal cell death [8]. Due to concerns that inhibition at this level would result in an overall negative effect of multiple intracellular pathways, more specific therapies, focused on specific downstream targets, may be less toxic and more beneficial.

Stimulation of the PI3-Akt pathway is well documented in ovarian, breast and colon cancer $[9,10]$. Limiting Akt activation suppresses gastrointestinal carcinoid and small cell lung cancer growth $[10,11]$. The Ras/Raf/MEK/ ERK mitogen-activating protein (MAP) kinase pathway plays a similar dual role, in melanoma and colon and lung cancers, acting as an oncogene, and as a tumor suppressor in NETs [12-15]. More recently, the role of insulin-like growth factor-1 (IGF-1) and its receptor (IGF-1R) in the development of NETs is being elucidated [16]. It is currently known that NETs express vascular endothelial growth factor (VEGF), epithelial growth factor (EGF) and their receptors, and IGF-1 and IGF-1R, which have all been noted to have an effect on tumor growth, invasion and motility via MEK and other intracellular pathways [17].

Another promising signaling pathway is Notch. Notch is a transmembrane protein that has been studied extensively in multiple malignancies. The isoform Notch-1 has been shown to act both as an oncogene and as a tumor suppressor. It has a role as an oncogene in pancreatic, colon and non-small cell lung cancers, and a tumor suppressor role in NETs, including pancreatic carcinoid tumors, medullary thyroid cancer (MTC), and small cell lung cancer $[8,18-23]$. The role of other Notch isoforms in cancer progression is still being investigated. Table 1 summarizes specific pharmacological agents that have an effect in each pathway.

The aim of this study is to review the intracellular pathways that are studied in NETs while focusing on specific targets that may serve as potential pharmacologic therapies. Current clinical trials with the use of drugs for the different pathways are discussed in the following text and summarized in table 1.

\section{RET Proto-Oncogene}

The RET proto-oncogene is a major focus of the intracellular pathways that determine the morphologic outcome of neuroendocrine tissues. It is the common denominator that transmits extracellular signals and thus affects multiple intracellular pathways [24]. RET has been thoroughly studied and its role is best known in MTC [25, 26]. However, its exact role in other NETs has yet to be completely elucidated.

Point mutations which lead to a gain of function in the RET tyrosine kinase have been noted in cases of multiple endocrine neoplasia (MEN) $2 \mathrm{~A}$ and $2 \mathrm{~B}$ and familial MTC; however, more information regarding the intracellular mechanism(s), is still needed $[25,26]$. This gene with 29 exons is located on chromosome 10q11.2 and encodes for a tyrosine kinase [26]. Alternate splicing results in the two intracellular tyrosine kinase domains stimulating several intracellular signal transduction pathways via three isoforms (RET 9, 43, and 51) known as the short, medium, and long domains (fig. 1, 2) [24]. Present on these isoforms are binding regions for glial cell-linederived neurotrophic factor, neurtorin, artemin, and persephin, which are located on one of four receptors (growth factor receptor $\alpha 1,2,3$, or 4) [27-32].

The knowledge that accumulated on the role of RET in endocrine tumors has led to a change in clinical practice. Genetic testing for RET oncogene mutations has proven key in the advancement of the treatment of MEN2A gene carriers presenting with MTC. As a result of their haplotype analysis, prophylactic thyroidectomy proved curative and provided insight into the nodal status in relation to the timing of intervention [33]. 
Table 1. Pharmacologic therapies, the intracellular pathway affected, their specific targets, and clinical trial status

\begin{tabular}{llll}
\hline Pathway & Drug & Mechanism & Clinical trial \\
\hline RET & Vandetanib (ZD6474) & Tyrosine kinase, VEGR, EGFR inhibitor & Phase III \\
& Gleevac (ST1571) & Tyrosine kinase inhibitor & Phase II \\
& AMG706 & Tyrosine kinase inhibitor & Phase II \\
\hline PI3-Akt & Afinitor (everolimus) & mTOR inhibitor & RADIANT-3 \\
& BEZ-235 & Torc 1\&2 inhibitor & N/A \\
& Lithium & GSK3 inhibitor & N/A \\
\hline Ras/Raf-1 & RAF265 & Raf-1 inhibitor & N/A \\
& Teriflunomide & Ras/Raf-1 inhibitor & N/A \\
& ZM336372 & Raf activator & N/A \\
& & GSK3 inhibitor & SUN1111 \\
& Sutent (SU11248) & Tyrosine kinase, PDGFR, VEGFR inhibitor & Phase III \\
& Gefitinib & EGFR & Phase II \\
& Bevacuzimab & VEGF-A antibody & Multi-center \\
& & & Phase III \\
\hline Notch & Suberoyl bis-hydroxamic acid & Histone deacetylase inhibitor & N/A \\
& Valproic acid & Notch-1 activator; HDAC inhibitor & Pilot phase II \\
\hline
\end{tabular}

Tyrosine kinase inhibitors target this NET common denominator to give a general non-specific inhibition of multiple downstream end products. XL184 and ZD6474 (vandetanib) target RET, resulting in suppression of cell proliferation and phosphorylation of RET and ERK, and inhibition of EGF and VEGF receptor (VEGFR) kinases, in vitro and in vivo [34-36]. Other tyrosine kinase inhibitors, including ST1571 (Gleevac) and AMG706, have also been shown to inhibit MTC cell growth in vitro and have undergone limited phase II clinical trials for hereditary MTC, with almost immediate reductions in calcitonin levels [37, 38]; however their role as a systemic therapy for decreasing tumor burden has yet to be demonstrated. Other tyrosine kinase inhibitors, which target the RET oncogene, work via the VEGF, EGF, or Raf-1/MEK/ ERK pathways [39-42]. Some success has been seen in disease stabilization, with vandetanib in stage II clinical trials for MTC; however as a whole, the results have not proven promising for metastatic disease [43]. Gefitinib, an EGFR tyrosine kinase inhibitor, blocks the anti-apoptotic Ras signal transduction cascade, and is showing some success in phase II clinical trials of patients with advanced thyroid cancers, including papillary, anaplastic and medullary variants [44].
Additional investigation into the role of the downstream targets of RET in familial and sporadic MTC, as well as carcinoid tumors, may further elucidate more specific therapeutic targets.

\section{PI3-Akt Pathway}

The PI3-Akt pathway is important in cell motility, proliferation and survival $[9,10]$. The focal points of these lipid kinases are the $\mathrm{p} 85$ and $\mathrm{p} 110$ subunits, which catalyze the conversion of phosphotidylinositol 4,5-bisphosphate to phosphotidylinositol 3,4,5-triphospate (fig. 1). Phosphotidylinositol 3,4,5-triphosphate plays a key role in activating Akt, a serine/threonine kinase. Akt, in turn, affects multiple downstream targets, including glycogen synthase kinase- $3 \beta$ (GSK3 $\beta$ ), nuclear transcription factor $\kappa \mathrm{B}(\mathrm{NF \kappa \textrm {B }})$, and mammalian target of rapamycin (mTOR) [45]. These genes have been shown to be involved in the progression of various cancers. The Akt1 isoform has been noted in multiple cancers, including human pancreatic carcinoid tumors, and works through mTOR, GSK3 $\beta$ and NFкB activation $[9,10,46]$. Mutations in the $\mathrm{p} 85$ or p110 subunits lead to loss of function in the phosphatase and tensin homolog (PTEN) leads to 
Fig. 1. Illustration of the RET, PI3K/Akt, Ras/Raf-1, and growth factors, intracellular pathways and pharmacological agents involved in NETs.
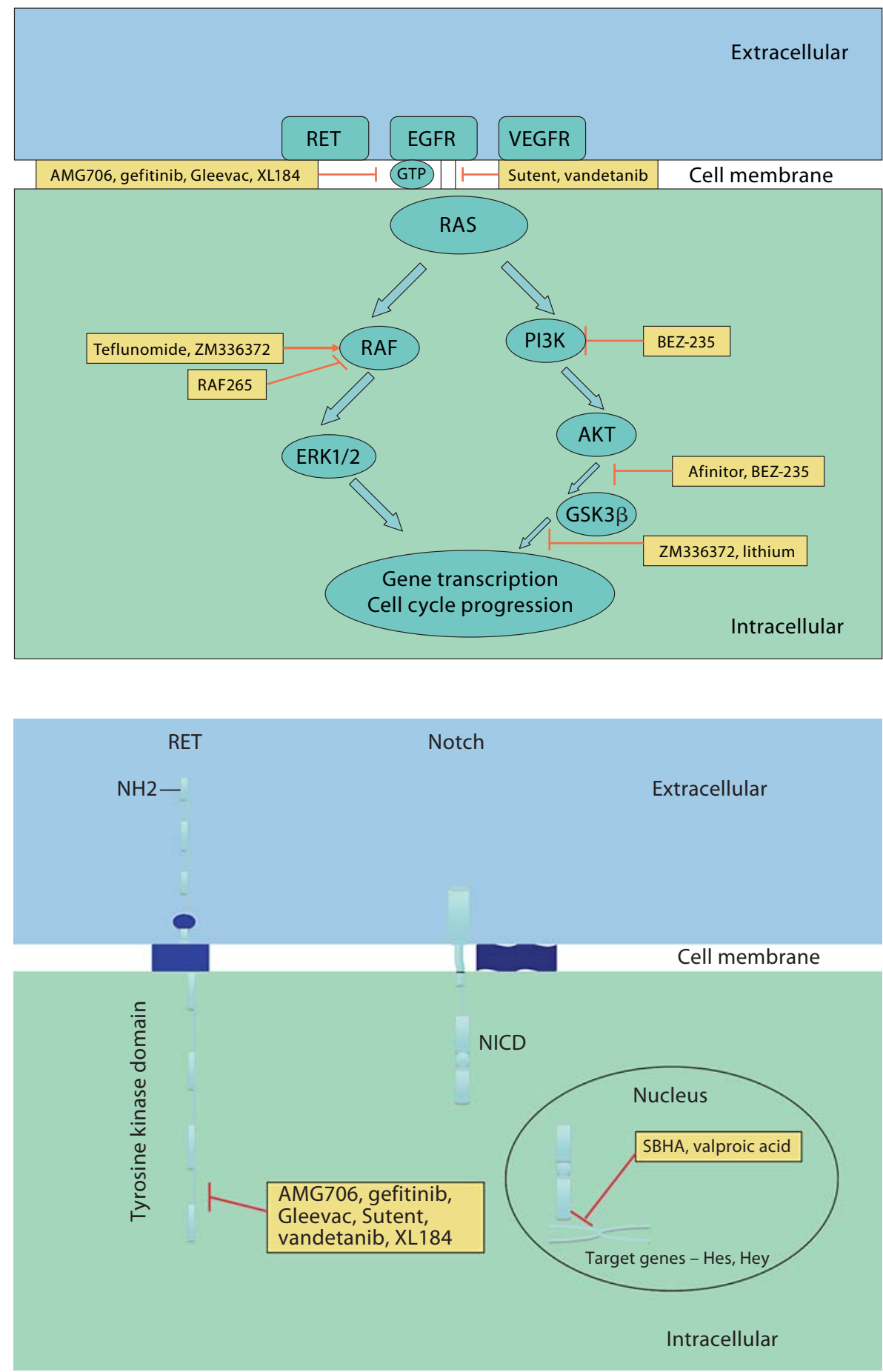

Fig. 2. Illustration of the RET and Notch intracellular pathways and pharmacological agents involved in NETs.
Phosphotidylinositol kinase (PI3K) can be activated by various integrins, tyrosine kinases, and B- and T-cell receptors. It subsequently acts as a docking protein for Akt. Akt then directly acts on p21 and p27, and indirectly on cyclin D1 and p53, via mTOR, to effect cell growth 
and pro-apoptotic signals $[48,49]$. Phosphotidylinositol is activated by RET tyrosine kinase 1062 , which has a binding site for phospholipase C $\gamma$ (PLC $\gamma$ ), as well as for multiple docking proteins (including for activation of ERK, p38 and JNK MAP kinases). The tyrosine kinase 1062-Akt pathway is important in NFKB activation [47] and pancreatic and pulmonary carcinoid cell survival $[50,51]$.

The multiple isoforms of Akt also have various activation pathways. Akt activation can be independent of, or dependent on PI3. Irrespective of its stimulation method, it plays critical event in nerve tissue generation; hence this may be yet another therapeutic target $[48,50]$. Investigations of this pathway have elucidated how Akt signaling in both gastrointestinal and pulmonary carcinoid tumors suppresses both cell proliferation and tumor marker expression, and induces apoptosis [18, 50, 52, 53]. In addition, Akt over activation plays a critical role in the pathogenesis of MTC and MEN2A and MEN2B RET oncogene expression $[52,53]$.

Several effective agents in this pathway have already been used, and show some promise as therapeutic targets in NETs, including lithium and ZM336372 which altered some of the downstream products, including GSK3 $\beta$, achaete-scute complex homolog-1 (ASCL1), and CgA [54-56]. Everolimus, an mTOR inhibitor, received Federal Drug Administration (FDA) approval in February 2011, after undergoing a successful phase III clinical trial, in which it attenuated disease progression and increased progression free survival in patients presenting with advanced pancreatic NETs [57]. Two recent studies have noted some success in the treatment of advanced MTC, utilizing dual therapy with medications that cross-talk amongst the intracellular pathways. A clinical trial comparing ZD6474, a RET, VEGFR and EGFR inhibitor, to placebo, after two successful phase II trials was just recently completed, and noted a significant improvement in progression-free survival in those patients with MTC [58]. Preclinical studies of RAF265 and BEZ-235, which inhibit the Raf and PI3K and target of rapamycin complex 1 (Torc1) and target of rapamycin complex 2 (Torc2) pathways, respectively, noted a synergistic effect, via blockage of both the ERK and PI3K signaling pathways, resulting in an attenuation of MTC growth [59].

Another signaling pathway downstream of Akt is the GSK $3 \beta$, a serine/threonine protein kinase. This pathway can be activated by phosphorylation by either the Akt or MAP kinase pathway. The tuberous sclerosis complex 2 (TSC2) gene is subsequently activated, and in turn, prevents the inhibition of the mTOR complex-1 protein, re- sulting in attenuation of cell growth, proliferation, angiogenesis and mitochondrial metabolism. Cell cycle arrest results via inhibition of cyclin D1 [60]. NFKB is a dimeric transcription factor which translocates into the nucleus to induce gene transcription and ultimately leads to cell survival and proliferation, after being released by phosphorylated inhibitor $\kappa B$ (IкB) or activated by GSK3 $\beta$. The activation of IкB is a result of Akt stimulation of $І \kappa B$ kinase. Inhibitors of this downstream target were initially thought of as potential therapies for diabetes, due to its role in glucose metabolism. However, further elucidation of the specific inhibition of its ser-9 position noted regulation of apoptosis and the cell cycle in NETs is needed. Afinitor (everolimus; Novartis Pharmaceuticals Co., East Hanover, N.J., USA) is an mTOR inhibitor. The phase III clinical trial, RAD001 in Advanced NETs (RADIANT)-3, resulted in a reduction of the risk of cancer progression and an improvement in progression-free survival in patients with progressive pancreatic NETs [57]. These more recent studies suggest a focus towards multiple downstream targets. The concerns to date are with tumor stabilization, side effects, and the therapeutic modality (neoadjuvant therapy, adjuvant therapy with surgery, or with a somatostatin analog).

\section{Ras/Raf-1/MEK/ERK1/2 Pathway}

Upon activation, Ras binds Raf- 1 at both the Ras-binding domain and the cysteine-rich domain, resulting in activation at four inducible sites (Ser 338, Tyr 341, Thr 491, and Ser 494). Phosphorylation of these activation sites results in activation of the MAPK/ERK1 kinasel and 2 (MEK1 and MEK2), via phosphorylation of Ser 217 and Ser 221. ERK1 and ERK2, which are 44 and $42 \mathrm{kDa}$ respectively, can then be phosphorylated and become active. Phosphorylation of all sites (Thr 202/Tyr 204 for ERK1 and Thr 185/Tyr 187 for ERK2) is required for full activation of ERK. Complete elucidation of all of the docking proteins involved and their activation of the MAP kinases is still being investigated $[7,13]$.

This pathway can be targeted upstream by inducing Ras mutations, which impair guanine triphosphatase activity, by stabilizing guanine triphosphate in the bound state, or more downstream, where ERK1 and ERK2 affect gene transcription (fig. 1). It is known that ERK regulates growth factor-responsive targets in the cytosol and regulates gene expression after translocating into the nucleus. It also indirectly phosphorylates protein $\mathrm{S6}$ kinase, which regulates mRNA transcription. Nuclear translocation is 
critical for gene expression and DNA replication, making this pathway a therapeutic target for cancer therapy.

Activation of the Raf-1/MEK/ERK pathway may be a therapeutic target for neuroendocrine cancers. Sippel et al. [12] demonstrated that activation of this pathway is associated with a reduction in neuroendocrine hormone production. Ectopic raf-1 expression leads to MTC growth suppression both in vitro and in vivo [55, 61]. Others have also demonstrated the role of this pathway in MTC. Ning et al. [61] noted its regulation of cell-cell contact molecules and its association with a metastatic phenotype in MTC. Increased Raf-1 activity, in gastrointestinal and pulmonary carcinoid tumor cell lines, at the transcriptional level, resulted in increased ERK1/2 phosphorylation and activation, with a decrease in NE hormone production, after treatment with ZM336372, a specific Raf activator [55, 56]. Raf-1 overexpression after MTC treatment with ZM336372 resulted in growth suppression and morphologic differentiation of the cells, which more closely resemble normal $\mathrm{C}$ cells $[62,63]$. This treatment is of interest as it has been shown to decrease bioactive hormone levels and expression of the transcription factor ASCL1 via upregulation and phosphorylation of Raf/MEK/ERK1/2 and suppress cell proliferation and induce cell cycle inhibitors p21 and p18. On the horizon are further, more specific therapies as a result of the decreased MTC cell viability noted $[64,65]$.

Pancreatic carcinoid cells (BON) have also been noted to have morphologic changes, including sharper boarders and a flatter shape, mimicking cellular differentiation, and representing the non-carcinogenic phenotype, with the activation of raf- 1 and its downstream target, ERK1/2 [64]. As a result of this pathway's role in gastrointestinal and pulmonary carcinoid tumors, as well as MTC, it is a reasonable therapeutic target. Teriflunomide, a novel Raf-1 pathway activator, has recently been proven to inhibit gastrointestinal carcinoid cell proliferation and decrease production of the tumor marker ASCL1 via inhibition of the MAPK kinase, activation of Raf-1/MEK and ERK1/2 and induction of G2-M arrest [65].

\section{Growth Factors}

Further downstream targets of the RET pathway, which could be more specific targets, are growth factors. NETs are highly vascularized and depend on growth factors which affect tumor cells, as well as endothelial cells, for survival. Several pro-angiogenic factors are overexpressed in NETs. VEGF, its receptors, and related signal- ing pathway components, including IGF-1, IGF-1R, EGF and hepatocyte growth factor (HGF), may also play role in vascular invasion, NET growth and metastases. The role of these growth factors is still being elucidated in NET growth and invasion. IGF-1 is a 70-amino-acid hormone, with a receptor (IGF-1R) that is a member of the tyrosine kinase super-family, with $70 \%$ homology to the insulin receptor [66]. This hormone has a role in differentiation, transformation and the prevention of apoptosis $[67,68]$. Two cascades have been identified in which this hormone utilizes, to effect tumor growth, VEGF expression and tumor invasion $[68,69]$. Cell survival is mediated via the PI3K, protein kinase B (PKB), GSK3 $\beta$, $\beta$ katenin and Myc-TCT 4 protein cascade; while the RasRaf-MAPK pathway regulates cell proliferation (fig. 1). The expression of IGF-1 and/or IGF-1R, as well as HGF, in NETs has been associated with advanced stage, increased size, poor prognosis/survival, and recurrence or metastases [16, 17, 70-72].

This pathway is a downstream target of at least three other intracellular pathways; hence it allows the option of a more specific therapeutic target. IGF-1-mediated neuroendocrine product regulation can be attenuated by stimulation of the raf-1/MEK1 pathway, inhibition of its autocrine loop, or as a result of stimulation of the Ras/PI3K/AKT system. Manipulation of this pathway, via stimulation of the PI3/AKT pathway, effects human BON NET cell survival and ultimate neuropeptide production, via alteration of cyclin D1 expression and increases CgA secretion.

EGF and HGF play key roles in tumor growth, aggressiveness, recurrence, motility and invasion [73-76]. These are important as overexpression of their receptors has been associated with increased tumor size, lymph node metastases, and poor prognosis/survival [77-84]. Nilsson et al. [84] demonstrated the role of EGFR in pheochromocytoma and MTC, as blockade with a monoclonal antibody, decreased tumor growth. Sutent (sunitinib; Pfizer, New York, N.Y., USA) is the first anti-VEGFR tyrosine kinase inhibitor approved. The SUN 111 phase III study resulted in a significant improvement in progression-free survival in patients with progressive, well-differentiated pancreatic NETs [85]. The results stem from the simultaneous inhibition of receptors for platelet-derived growth factor and VEGF, which reduced tumor vascularization and increased cancer cell death and tumor shrinkage. Another drug which may prove more promising in combination with other drugs is bevacizumab. This monoclonal VEGF-A antibody is currently in phase III clinical trials after demonstrating improved progression-free 
survival and attenuated tumor perfusion and progression in a phase II clinical trial of patients with advanced carcinoid tumors [86].

\section{NOTCH-1/Achaete-Scute Complex-Like 1 (ASCL1)}

This highly conserved pathway plays an important role in embryonic development. It maintains stem cells, influences the final fate of cells, and generates terminal differentiation processes $[20,87,88]$. Notch proteins are comprised of four $300-\mathrm{kDa}$ transmembrane receptors and five ligands. After stimulation via cell-to-cell contact and ligand binding (DLL 1, 3, 4 or Jagged 1,2), a sequence of proteolytic cleavages occurs, with the subsequent activation of the Notch intracellular domain (fig. 2) [87, 88]. This domain translocates into the nucleus and interacts with CBF-1/RBPjk resulting in the activation of various genes, including the suppressor of hairless and Lag-1 (HES-1). Notch-1 has been identified as both an oncogene and a tumor suppressor. It was first noted to stimulate cell proliferation and attenuate apoptosis in T-cell acute lymphocytic leukemia, breast cancer, melanoma, non-small cell lung cancer, and the renal epithelial cell $[20,87,88]$. Its role as a tumor suppressor was noted in keratinocytes and astrocytomas, with a loss of function of Notch-1 resulting in a negative regulation of tumorigenesis [46]. More recent research has noted a fine balance which must be adhered to with regard to Notch expression. Excessive up- or downregulation can be detrimental $[87,88]$.

The role of the Notch pathway in NETs appears to be opposite of that in epithelial-derived cancers. Overexpression of Notch inhibits cell proliferation and induces apoptosis, rather than promoting the growth of these tumors $[22,23,89]$. VEGF induces Notch-1 and $\delta$-like4 expression via activation of the PI3K-Akt pathway, and elevated Jagged 1 expression, in tumors, increases Notch-1 activation in endothelial cells, resulting in vascular network formation and hence vascular supply to tumors. Notch activation suppresses MTC and carcinoid tumor growth and hormone production [89-93]. This pathway is conserved in gastrointestinal and pulmonary carcinoid tumors. Additional studies of this pathway have noted attenuation of ASCL1 production and modulation of the neuroendocrine phenotype in carcinoid tumors, associated with Notch expression [22]; hence it is a novel therapeutic target to exploit. Further studies have shown that downregulation of ASCL1 transcription and growth suppression of NETs by Notch is HES-1-depen- dent $[22,89]$. In vitro studies with valproic acid and histone deacetylase (HDAC) inhibitors proved beneficial in inducing apoptosis and decreasing cell growth in papillary, follicular, papillary and MTCs, as well as carcinoid tumors, via alterations in Notch expression [89-91]. A recent phase II pilot study utilizing valproic acid, to induce Notch-1 expression, noted its efficacy in the treatment of low-grade neuroendocrine carcinoma [92]. This supported a previous in vivo study in which the expression of active Notch-1 correlated with a decrease in MTC growth [51].

This multifunctional transmembrane receptor also plays a role in cell differentiation, proliferation and survival $[20,22,87]$. Noted to be absent or have minimal expression in NETs, Notch-1 activation and expression has been shown to be a tumor suppressor in MTC and carcinoid tumors, resulting in a reduction of both in vivo and in vitro tumor growth, as well as attenuation of the production of neuropeptides $\mathrm{CgA}$, serotonin neuron-specific enolase and synaptophysin, and the tumor marker ASCL1 $[90,93]$. Due to its multiple binding sites, more than one pathway is probably involved in the generation of NETs, especially MTC, as some mutations are evident with more aggressive phenotypes. Hence, the Notch pathway is a prime target for new, unconventional pharmacologic therapeutic agents. Valproic acid and suberoyl bis-hydroxamic acid, HDAC inhibitors, have been demonstrated both in vitro and in vivo, to upregulate Notch-1 signaling and suppress growth in papillary thyroid cancer, follicular thyroid cancer, MTC, and carcinoid cells $[45,93]$. In addition, ASCL1 expression and cyclin D1 were suppressed, and $\mathrm{p} 21$ production was increased. Recently reported phase II clinical trial results from patients treated with valproic acid are promising. Patients with low-grade carcinoid or pancreatic NETs had upregulation of Notch-1 signaling, and demonstrated either a partial tumor response or stable disease [92]. With more knowledge of the downstream targets, both the individual isoforms and downstream effectors can be targeted for more specific pathway inhibition. Another area beginning to be focused on is combination therapy. Adler et al. [94] recently concluded an in vitro study of carcinoid tumors treated with lithium and HDAC inhibitors, demonstrating tumor growth suppression. With more targets and combination therapy, specific downstream effectors can be targeted, and toxicity and side effects can be minimized. 


\section{Conclusion}

There are several pathways which regulate the proliferation of neuroendocrine cancers. However, further elucidation of these pathways may hold the key to not only improvement and resolution of symptoms, but inhibition of tumor growth. The downstream targets in these pathways are providing more therapeutic options, as well as more information regarding the pathogenesis of these tumors. By having more specific targets, treatments can be tailored to appropriate tumors in order to enhance effec- tiveness and minimize toxicity and side effects. Some current ongoing clinical trials attempting to target these specific pathways show encouraging results and offer hope to the patients who otherwise have limited other therapies available.

\section{Acknowledgements}

We thank Nicholas Yeutter for his contribution to the data search for this review. This study was funded by NIH/NCI Supplemental Grant RO1CA121115-S1.

\section{References}

$\checkmark 1$ Rindi G, Wiedenmann B: Neuroendocrine 12 Sippel RS, et al: The role of human achaeteneoplasms of the gut and pancreas: new in- scute homolog-1 in medullary thyroid cansights. Nat Rev Endocrinol 2011;8:54-64.

2 Moller JE, et al: Factors associated with progression of carcinoid heart disease. N Engl J Med 2003;348:1005-1015.

3 Chan JA, Kulke MH: New treatment options for patients with advanced neuroendocrine tumors. Curr Treat Options Oncol 2011;12: $136-148$.

4 Oberg KE, et al: Role of somatostatins in gastroenteropancreatic neuroendocrine tumor development and therapy. Gastroenterology 2010;139:742-753, $753 \mathrm{el}$.

5 Oberg K: Cancer: antitumor effects of octreotide LAR, a somatostatin analog. Nat Rev Endocrinol 2010;6:188-189.

6 Rinke A, et al: Placebo-controlled, doubleblind, prospective, randomized study on the effect of octreotide LAR in the control of tumor growth in patients with metastatic neuroendocrine midgut tumors: a report from the PROMID Study Group. J Clin Oncol 2009;27:4656-4663.

7 Ichihara M, Murakumo Y, Takahashi M: RET and neuroendocrine tumors. Cancer Lett 2004;204:197-211.

8 Ye L, Santarpia L, Gagel RF: The evolving field of tyrosine kinase inhibitors in the treatment of endocrine tumors. Endocr Rev 2010;31:578-599.

9 Luo J, Manning BD, Cantley LC: Targeting the PI3K-Akt pathway in human cancer: rationale and promise. Cancer Cell 2003;4: 257-262.

10 Vivanco I, Sawyers CL: The phosphatidylinositol 3-kinase AKT pathway in human cancer. Nat Rev Cancer 2002;2:489-501.

-11 Krystal GW, Sulanke G, Litz J: Inhibition of phosphatidylinositol 3-kinase-Akt signaling blocks growth, promotes apoptosis, and enhances sensitivity of small cell lung cancer cells to chemotherapy. Mol Cancer Ther 2002;1:913-922. cer cells. Surgery 2003;134:866-873.

13 Kunnimalaiyaan M, Chen H: The Raf-1 pathway: a molecular target for treatment of select neuroendocrine tumors? Anticancer Drugs 2006; 17:139-142.

14 Greco A, et al: Molecular pathology of differentiated thyroid cancer. Q J Nucl Med Mol Imaging 2009;53:440-453.

15 Walker GJ, Hayward NK: Pathways to melanoma development: lessons from the mouse. J Invest Dermatol 2002;119:783-792.

16 Von Wichert G, et al: Insulin-like growth factor-I is an autocrine regulator of chromogranin A secretion and growth in human neuroendocrine tumor cells. Cancer Res 2000;60:4573-4581.

17 Peters G, et al: IGF-1R, IGF-1 and IGF-2 expression as potential prognostic and predictive markers in colorectal-cancer. Virchows Arch 2003;443:139-145.

18 Pitt SC, Chen H, Kunnimalaiyaan M: Inhibition of phosphatidylinositol 3-kinase/Akt signaling suppresses tumor cell proliferation and neuroendocrine marker expression in GI carcinoid tumors. Ann Surg Oncol 2009; 16:2936-2942.

19 Murtaugh LC, et al: Notch signaling controls multiple steps of pancreatic differentiation. Proc Natl Acad Sci USA 2003;100:1492014925.

20 Kadesch T: Notch signaling: the demise of elegant simplicity. Curr Opin Genet Dev 2004; 14:506-512.

21 Radtke F, Raj K: The role of Notch in tumorigenesis: oncogene or tumour suppressor? Nat Rev Cancer 2003;3:756-767.

22 Kunnimalaiyaan M, Traeger K, Chen $\mathrm{H}$ : Conservation of the Notch-1 signaling pathway in gastrointestinal carcinoid cells. Am J Physiol Gastrointest Liver Physiol 2005; 289:G636-G642.
23 Nakakura EK, et al: Regulation of neuroendocrine differentiation in gastrointestinal carcinoid tumor cells by Notch signaling. J Clin Endocrinol Metab 2005;90:4350-4356.

24 Takahashi M, Ritz J, Cooper GM: Activation of a novel human transforming gene, ret, by DNA rearrangement. Cell 1985;42:581-588.

25 Donis-Keller $\mathrm{H}$, et al: Mutations in the RET proto-oncogene are associated with MEN2A and familial medullary thyroid cancer. Hum Mol Genet 1993;2:851-856.

26 Mulligan LM, et al: Germ-line mutations of the RET proto-oncogene in multiple endocrine neoplasia type 2A. Nature 1993;363: 458-460.

27 Myers SM, et al: Characterization of RET proto-oncogene $3^{\prime}$ splicing variants and polyadenylation sites: a novel C-terminus for RET. Oncogene 1995;11:2039-2045.

28 Lin LF, et al: GDNF: a glial cell line-derived neurotrophic factor for midbrain dopaminergic neurons. Science 1993;260:11301132.

29 Kotzbauer PT, et al: Neurturin, a relative of glial-cell-line-derived neurotrophic factor. Nature 1996;384:467-470.

30 Baloh RH, et al: Artemin, a novel member of the GDNF ligand family, supports peripheral and central neurons and signals through the GFR $\alpha 3$-RET receptor complex. Neuron 1998;21:1291-1302.

31 Milbrandt J, et al: Persephin, a novel neurotrophic factor related to GDNF and neurturin. Neuron 1998;20:245-253.

- 32 Airaksinen MS, Titievsky A, Saarma M: GDNF family neurotrophic factor signaling: four masters, one servant? Mol Cell Neurosci 1999;13:313-325.

33 Moley JF: Medullary thyroid cancer. Surg Clin North Am 1995;75:405-420.

34 Verbeek HH, et al: The effects of four different tyrosine kinase inhibitors on medullary and papillary thyroid cancer cells. J Clin Endocrinol Metab 2011;96:E991-E995. 
-35 Carlomagno F, et al: Disease associated mutations at valine 804 in the RET receptor tyrosine kinase confer resistance to selective kinase inhibitors. Oncogene 2004;23:60566063.

-36 Vitagliano D, et al: The tyrosine kinase inhibitor ZD6474 blocks proliferation of RET mutant medullary thyroid carcinoma cells. Endocr Relat Cancer 2011;18:1-11.

-37 Cohen MS, Hussain HB, Moley JF: Inhibition of medullary thyroid carcinoma cell proliferation and RET phosphorylation by tyrosine kinase inhibitors. Surgery 2002;132: 960-967.

-38 Sippel RS, Kunnimalaiyaan M, Chen H: Current management of medullary thyroid cancer. Oncologist 2008;13:539-547.

39 Wedge SR, et al: ZD6474 inhibits vascular endothelial growth factor signaling, angiogenesis, and tumor growth following oral administration. Cancer Res 2002;62:46454655.

40 Kim S, et al: Sorafenib inhibits the angiogenesis and growth of orthotopic anaplastic thyroid carcinoma xenografts in nude mice. $\mathrm{Mol}$ Cancer Ther 2007;6:1785-1792.

-41 Kim DW, et al: An orally administered multitarget tyrosine kinase inhibitor, SU11248, is a novel potent inhibitor of thyroid oncogenic RET/papillary thyroid cancer kinases. J Clin Endocrinol Metab 2006;91:40704076.

$\checkmark 42$ Hoelting $\mathrm{T}$, et al: Epidermal growth factor enhances proliferation, migration, and invasion of follicular and papillary thyroid cancer in vitro and in vivo. J Clin Endocrinol Metab 1994;79:401-408.

$\checkmark 43$ Wells SA Jr, et al: Vandetanib for the treatment of patients with locally advanced or metastatic hereditary medullary thyroid cancer. J Clin Oncol 2010;28:767-772.

44 Pennell NA, et al: A phase II study of gefitinib in patients with advanced thyroid cancer. Thyroid 2008;18:317-323.

-45 Zarebczan B, Chen H: Signaling mechanisms in neuroendocrine tumors as targets for therapy. Endocrinol Metab Clin North Am 2010;39:801-810.

-46 Somasundaram K, et al: Upregulation of ASCL1 and inhibition of Notch signaling pathway characterize progressive astrocytoma. Oncogene 2005;24:7073-7083.

$\checkmark 4$ Hayashi H, et al: Characterization of intracellular signals via tyrosine 1062 in RET activated by glial cell line-derived neurotrophic factor. Oncogene 2000;19:4469-4475.

$\checkmark 48$ De Vita G, et al: Tyrosine 1062 of RETMEN2A mediates activation of Akt (protein kinase B) and mitogen-activated protein kinase pathways leading to PC12 cell survival. Cancer Res 2000;60:3727-3731.

$\checkmark 49$ Van Weering DH, Bos JL: Glial cell line-derived neurotrophic factor induces Ret-mediated lamellipodia formation. J Biol Chem 1997;272:249-254.
50 Pitt SC, Chen H, Kunnimalaiyaan M: Phosphatidylinositol 3-kinase-Akt signaling in pulmonary carcinoid cells. J Am Coll Surg 2009;209:82-88.

51 Pitt SC, et al: AKT and PTEN expression in human gastrointestinal carcinoid tumors. Am J Transl Res 2009; 1:291-299.

52 Pitt SC, Chen H: The phosphatidylinositol 3-kinase/akt signaling pathway in medullary thyroid cancer. Surgery 2008;144:721724 .

53 Kunnimalaiyaan M, Ndiaye M, Chen $\mathrm{H}$ : Apoptosis-mediated medullary thyroid cancer growth suppression by the PI3K inhibitor LY294002. Surgery 2006;140:1009-1015.

54 Greenblatt DY, et al: Lithium inhibits carcinoid cell growth in vitro. Am J Transl Res 2010;2:248-253.

55 Van Gompel JJ, et al: ZM336372, a Raf-1 activator, suppresses growth and neuroendocrine hormone levels in carcinoid tumor cells. Mol Cancer Ther 2005;4:910-917.

56 Kunnimalaiyaan M, Ndiaye M, Chen H: Neuroendocrine tumor cell growth inhibition by ZM336372 through alterations in multiple signaling pathways. Surgery 2007; 142:959-964.

57 Yao JC, et al: Everolimus for advanced pancreatic neuroendocrine tumors. N Engl J Med 2011;364:514-523.

58 Deshpande H, et al: Vandetanib (ZD6474) in the treatment of medullary thyroid cancer Clin Med Insights Oncol 2011;5:213-221.

59 Jin N, et al: Synergistic action of a RAF inhibitor and a dual PI3K/mTOR inhibitor in thyroid cancer. Clin Cancer Res 2011;17: 6482-6489.

60 Van Weering DH, Bos JL: Signal transduction by the receptor tyrosine kinase Ret. Recent Results Cancer Res 1998;154:271-281.

61 Ning L, Kunnimalaiyaan M, Chen H: Regulation of cell-cell contact molecules and the metastatic phenotype of medullary thyroid carcinoma by the Raf-1/MEK/ERK pathway. Surgery 2008; 144:920-925.

62 Vaccaro A, Chen H, Kunnimalaiyaan M: Invivo activation of Raf-1 inhibits tumor growth and development in a xenograft model of human medullary thyroid cancer. Anticancer Drugs 2006;17:849-853.

63 Chen H, et al: Differentiation of medullary thyroid cancer by C-Raf-1 silences expression of the neural transcription factor human achaete-scute homolog-1. Surgery 1996; 120:168-173.

64 Sippel RS, Chen H: Activation of the Ras/ Raf-1 signal transduction pathway in carcinoid tumor cells results in morphologic transdifferentiation. Surgery 2002;132: 1035-1039.

65 Cook MR, et al: Identification of a novel Raf1 pathway activator that inhibits gastrointestinal carcinoid cell growth. Mol Cancer Ther 2010;9:429-437.

66 Hakam A, et al: Expression of insulin-like growth factor-1 receptor in human colorectal cancer. Hum Pathol 1999;30:1128-1133.
67 Reinmuth N, et al: Impact of insulin-like growth factor receptor-I function on angiogenesis, growth, and metastasis of colon cancer. Lab Invest 2002;82:1377-1389.

68 Lopez T, Hanahan D: Elevated levels of IGF1 receptor convey invasive and metastatic capability in a mouse model of pancreatic islet tumorigenesis. Cancer Cell 2002;1:339-353.

69 Maiorano E, et al: Insulin-like growth factor-1 expression in thyroid tumors. Appl Immunohistochem Mol Morphol 2000;8:110119.

70 To CT, Tsao MS: The roles of hepatocyte growth factor/scatter factor and met receptor in human cancers. Oncol Rep 1998;5: 1013-1024.

71 Gydee H, et al: Differentiated thyroid carcinomas from children and adolescents express IGF-I and the IGF-I receptor (IGF-I-R). Cancers with the most intense IGF-I-R expression may be more aggressive. Pediatr Res 2004;55:709-715.

72 Woodburn JR: The epidermal growth factor receptor and its inhibition in cancer therapy. Pharmacol Ther 1999;82:241-250.

73 Maggiora P, et al: Control of invasive growth by the HGF receptor family. J Cell Physiol 1997; 173:183-186.

74 Di Renzo MF, et al: Expression of the Met/ HGF receptor in normal and neoplastic human tissues. Oncogene 1991;6:1997-2003.

75 Modlin IM, Sandor A: An analysis of 8,305 cases of carcinoid tumors. Cancer 1997;79: 813-829.

76 Chen BK, et al: Co-overexpression of p53 protein and epidermal growth factor receptor in human papillary thyroid carcinomas correlated with lymph node metastasis, tumor size and clinicopathologic stage. Int J Oncol 1999; 15:893-898.

77 Umeki K, Shiota G, Kawasaki H: Clinical significance of c-met oncogene alterations in human colorectal cancer. Oncology 1999;56: 314-321.

78 Camp RL, Rimm EB, Rimm DL: Met expression is associated with poor outcome in patients with axillary lymph node negative breast carcinoma. Cancer 1999;86:22592265.

79 Chen BK, et al: Overexpression of c-Met protein in human thyroid tumors correlated with lymph node metastasis and clinicopathologic stage. Pathol Res Pract 1999;195: 427-433.

80 Cortesina $\mathrm{G}$, et al: Staging of head and neck squamous cell carcinoma using the MET oncogene product as marker of tumor cells in lymph node metastases. Int J Cancer 2000; 89:286-292.

-81 Sawatsubashi M, et al: Expression of c-Met in laryngeal carcinoma. Virchows Arch 1998; 432:331-335.

82 Di Renzo MF, et al: Overexpression of the c$\mathrm{MET} / \mathrm{HGF}$ receptor in human thyroid carcinomas derived from the follicular epithelium. J Endocrinol Invest 1995;18:134-139. 
83 Hirose Y, et al: Clinical importance of c-Met protein expression in high-grade astrocytic tumors. Neurol Med Chir (Tokyo) 1998;38: 851-859.

84 Nilsson O, et al: Presence of IGF-I in human midgut carcinoid tumours - an autocrine regulator of carcinoid tumour growth? Int J Cancer 1992;51:195-203.

-85 Raymond E, et al: Sunitinib malate for the treatment of pancreatic neuroendocrine tumors. N Engl J Med 2011;364:501-513.

86 Yao JC, et al: Targeting vascular endothelial growth factor in advanced carcinoid tumor: a random assignment phase II study of depot octreotide with bevacizumab and pegylated interferon- $\alpha_{2 b}$. J Clin Oncol 2008;26:13161323.
87 Maillard I, Pear WS: Notch and cancer: best to avoid the ups and downs. Cancer Cell 2003;3:203-205.

88 Yoon K, Gaiano N: Notch signaling in the mammalian central nervous system: insights from mouse mutants. Nat Neurosci 2005;8:709-715.

89 Xiao X, Ning L, Chen H: Notch-1 mediates growth suppression of papillary and follicular thyroid cancer cells by histone deacetylase inhibitors. Mol Cancer Ther 2009;8: 350-356.
90 Greenblatt DY, et al: Valproic acid activates Notch-1 signaling and induces apoptosis in medullary thyroid cancer cells. Ann Surg 2008;247:1036-1040.

91 Greenblatt DY, et al: Valproic acid activates Notch-1 signaling and regulates the neuroendocrine phenotype in carcinoid cancer cells. Oncologist 2007;12:942-951.

92 Mohammed TA, et al: A pilot phase II study of valproic acid for treatment of low-grade neuroendocrine carcinoma. Oncologist 2011;16:835-843.

$\$ 93$ Jaskula-Sztul R, et al: Expression of the active Notch-1 decreases MTC tumor growth in vivo. J Surg Res 2011;171:23-27.

-94 Adler JT, et al: Combination therapy with histone deacetylase inhibitors and lithium chloride: a novel treatment for carcinoid tumors. Ann Surg Oncol 2009;16:481-486. 